\title{
Autoimmune autonomic neuropathies: time to look beyond autoimmune autonomic ganglionopathy
}

\author{
Srikanth Muppidi ${ }^{1}$
}

Received: 19 December 2017 / Accepted: 21 December 2017 / Published online: 5 January 2018

๑) Springer-Verlag GmbH Germany, part of Springer Nature 2018

Autoimmune autonomic neuropathies are rare disorders with an acute or subacute onset of autonomic failure which are potentially responsive to immunotherapy. Among these diseases, autoimmune autonomic ganglionopathy (AAG) is the most well-known syndrome. AAG is caused by antibodies against the ganglionic nicotinic acetylcholine receptor (AChR) that disrupt cholinergic transmission at the sympathetic and parasympathetic ganglia. Ganglionic AChR antibodies are known to have a pathogenic role in AAG [1], and antibody levels correlate with disease severity [2]. In addition, AAG has many unique features, including severe autonomic failure, pupillary fatigue, and responsiveness to known therapies for antibody-mediated diseases such as plasma exchange, intravenous immunoglobulins (IVIG), or other types of immunotherapy. Autonomic clinicians and neurologists are aware of AAG and usually have a fairly low threshold for requesting ganglionic $\mathrm{AChR}$ antibody titers in acute- or subacute-onset focal or generalized autonomic syndromes, since they are relatively easy to obtain [3]. However, not all patients with autoimmune autonomic neuropathies have a positive titer of ganglionic AChR antibodies. If the degree and nature of the autonomic neuropathy is severe (e.g., with associated severe orthostatic hypotension), and all signs point to an underlying autoimmune etiology, these seronegative patients are typically treated with immunotherapy too. In clinical practice, the treatment response and long-term outcomes of patients with acute-onset autonomic failure and negative ganglionic $\mathrm{AChR}$ antibodies are unknown.

In this issue of Clinical Autonomic Research, Drs. Golden, Bryarly, and Vernino present their experience in these patients with antibody-negative, albeit severe,

Srikanth Muppidi

muppidis@stanford.edu

1 Department of Neurology, Stanford Medical Center, Stanford Neuroscience Health Center, 213 Quarry Road, 2nd Floor, Palo Alto, CA 94304, USA autoimmune autonomic neuropathy [5]. This case series offers many novel useful insights into patients with seronegative autoimmune autonomic neuropathy. Clinically, seronegative patients seem to have a higher incidence of sensory symptoms with neuropathic pain. On autonomic testing, they seem to have predominant sympathetic impairment and do not exhibit the obvious pupillary fatigue reported in patients with AAG. More importantly, these patients do not seem to respond to therapies commonly used for antibody-mediated diseases, such as plasma exchange, IVIG, or rituximab. Instead, these seronegative patients appear to respond predominantly to high doses of steroids. The authors rightly propose that the underlying pathophysiology in these patients might be different from AAG, and propose a cellmediated or inflammatory immune process rather than an antibody-mediated mechanism.

This small case series of patients with severe autonomic neuropathy raises some questions. Because treatment response was assessed based on symptomatic improvement only, and since this is likely retrospectively reported, could there be some bias in recall or patient selection? Does the hypothesis of a non-antibody-mediated process apply only to patients with severe autonomic neuropathy, or is it relevant to all other forms of seronegative, mild or focal, autonomic neuropathies? Since this series included mostly young patients (4 out of 6 patients were younger than 40), would older patients show similar positive response to very high dose steroids?

Perhaps even more interesting is the similarity of these patients to those with acute autonomic and sensory neuropathy (AASN). AASN is a rare Guillain-Barré-like syndrome with no motor involvement that is predominantly described in subjects of Asian origin. It has been reported after infections and typically presents as an acute-onset syndrome with significant sensory deficits (including lack of pain and temperature sensation, lack of proprioception, and severe sensory ataxia) combined with neuropathic pain and severe autonomic failure (including orthostatic hypotension, atonic 
bladder, and severe gastroparesis). These patients also have characteristic hyperintensities in the dorsal columns on spinal magnetic resonance (MR) imaging as well as very low plasma norepinephrine levels [4]. Although the patients of this series reported by Golden, Bryarly, and Vernino seem to have a predominantly subacute course with no changes on spinal MR imaging, their clinical phenotypes are remarkably similar to those of patients with AASN [5]. Do these seronegative patients have more in common with AASN than with AAG, and does that provide clues to the underlying pathophysiology?

As with any new interesting work, there are as many questions as answers, and this augurs well for the field. It should be expected that this single-center retrospective series will be the prelude to a multicenter prospective observational study of similar patients in which treatment response is assessed objectively and biological samples are collected, as this remains the only way for us improve our understanding of these rare and debilitating yet potentially treatable syndromes.

Funding None.

\section{Compliance with ethical standards}

Conflict of interest The author declares that he has no conflict of interest.

\section{References}

1. Vernino S, Ermilov LG, Sha L, Szurszewski JH, Low PA, Lennon VA (2004) Passive transfer of autoimmune autonomic neuropathy to mice. J Neurosci 24(32):7037-7042. https://doi.org/10.1523/ JNEUROSCI.1485-04.2004

2. Gibbons CH, Freeman R (2009) Antibody titers predict clinical features of autoimmune autonomic ganglionopathy. Auton Neurosci 146(1-2):8-12. https://doi.org/10.1016/j.autneu.2008.11.013

3. Vernino S (2009) Antibody testing as a diagnostic tool in autonomic disorders. Clin Auton Res. https://doi.org/10.1007/s102 86-008-0488-6

4. Koike H, Atsuta N, Adachi H et al (2010) Clinicopathological features of acute autonomic and sensory neuropathy. Brain 133(10):2881-2896. https://doi.org/10.1093/brain/awq214

5. Golden EP, Bryarly MA, Vernino S (2017) Seronegative autoimmune autonomic neuropathy: a distinct clinical entity. Clin Auton Res. https://doi.org/10.1007/s10286-017-0493-8 